\title{
Changes in Trunk Variability and Stability of Gait in Patients with Chronic Low Back Pain: Impact of Laboratory versus Daily-Living Environments
}

\author{
Yuki Nishi ${ }^{1,2}$ \\ Hayato Shigetoh (ID) \\ Ren Fujii' \\ Michihiro Osumi ${ }^{1,3}$ \\ Shu Morioka (iD) ${ }^{1,3}$ \\ 'Department of Neurorehabilitation, \\ Graduate School of Health Science, Kio \\ University, Nara, Japan; ${ }^{2}$ Department of \\ Rehabilitation Medicine, Nishiyamato \\ Rehabilitation Hospital, Nara, Japan; \\ ${ }^{3}$ Neurorehabilitation Research Center, \\ Kio University, Nara, Japan
}

Correspondence: Yuki Nishi

Department of Neurorehabilitation Graduate School of Health Science, Kio University, 4-2-2 Umaminaka, Koryo-cho, Kitakatsuragi-gun, Nara, 635-0832, Japan

Tel +8I-74-554-|60I

Fax +8I-74-554-1600

Email y.nishi.pt@gmail.com
Background: Individuals with chronic low back pain (CLBP) experience changes in gait control due to pain and/or fear. Although CLBP patients' gait has been performed in laboratory environments, changes in gait control as an adaptation to unstructured daily living environments may be more pronounced than the corresponding changes in laboratory environments. We investigated the impacts of the environment and pathology on the trunk variability and stability of gait in CLBP patients.

Methods: CLBP patients $(n=20)$ and healthy controls with no low-back pain history $(n=20)$ were tasked with walking in a laboratory or daily-living environment while wearing an accelerometer on the low back. We calculated the stride-to-stride standard deviation and multiscale sample entropy as indices of "gait variability" and the maximum Lyapunov exponent as an index of "gait stability" in both the anterior-posterior and medial-lateral directions. The participants were assessed on the numerical rating scale for pain intensity, the Tampa Scale for Kinesiophobia, and the Roland-Morris Disability Questionnaire for quality of life (QOL).

Results: In a repeated-measures ANOVA, the standard deviation was affected by environment in the anterior-posterior direction and by group and environment in the medial-lateral direction. Multiscale sample entropy showed no effect in the anterior-posterior direction and showed both effects in the medial-lateral direction. Maximum Lyapunov exponents showed both effects in the anterior-posterior direction, but none in the medial-lateral direction. These changes of trunk motor control by CLBP patients were found to be related to pain intensity, fear of movement, and/or QOL in the daily-living environment but not in the laboratory environment.

Conclusion: These results revealed that CLBP patients exhibit changes in trunk variability and stability of gait depending on the environment, and they demonstrated that these changes are related to pain, fear, and QOL. We propose useful accelerometer-based assessments of qualitative gait in CLBP patients' daily lives, as it would provide information not available in a general practice setting.

Keywords: chronic low back pain, daily-living gait, variability index, stability index, inertial sensor

\section{Introduction}

Chronic low back pain (CLBP), which is one of the most common health problems, is the leading cause of limitations in gait and daily living activities. $^{1-3}$ Individuals with CLBP are known to have characteristic spatiotemporal gait parameters, including reduced step length, decreased cadence, and increased stride-to-stride variability. ${ }^{4-7}$ They also frequently present with impaired lumbar movements, such as a reduction in movement velocity and 
a decreased range of movement. ${ }^{8}$ In general, motor control is affected among individuals with CLBP in the way that Hodges proposed - ie, with changes in multiple levels of the motor system to protect the tissues from further pain or injury, such as changes in the pattern of activity of trunk muscles and mechanical behaviors, and reduced responsiveness to sensory input. ${ }^{9}$ Hodges further reported that pain and/or pain-related fear alter motor variability and trunk stabilization as motor system adaptations in individuals with CLBP. ${ }^{9}$ However, these adaptive phenomena of gait have not been clarified.

Gait variability is a reflection of the multiple degrees of freedom of the sensory-motor system. ${ }^{10}$ Both the standard deviation (SD), which is one of the classical linear estimates, and multiscale sample entropy (MSE), which is based on the quantification of complexity on multiple scales of time series, have been used to evaluate gait variability. ${ }^{11-13}$ Gait stability is considered the ability of an individual's sensory-motor control system to maintain a gait in the presence of omnipresent disturbances, and it is often evaluated by using the maximum Lyapunov exponent (LyE), which is a nonlinear measure of local dynamic stability. ${ }^{14,15}$ Uncovering the variability and the stability of the gait pattern could provide more insight into the behavioral changes in individuals with CLBP.

Previous studies employing a single wearable sensor to evaluate gait reported that the variability and/or stability of gait are more affected in a daily-living environment than in a laboratory or clinical environment. ${ }^{16-18}$ In particular, healthy young adults routinely vary their motor patterns to maintain stability in unstructured environments. ${ }^{18}$ Measuring gait in daily living reflects individuals' habitual gait performance, ${ }^{19,20}$ and thus the recording of the gait during daily living has clinical utility and presents the potential benefit of evaluating gaits under unsupervised and uncontrolled conditions. ${ }^{21,22}$ Moreover, gaits in unstructured daily living are more difficult to predict and thus more prone to disturbance than those measured in structured clinical settings.

We conducted the present study to use the measures of SD, MSE, and LyE to explore the impacts of the subjects' environment and pathology by analyzing differences in trunk variability and stability of gait between individuals with CLBP and healthy controls in both laboratory and daily-living environments. We also investigated the relationship between trunk variability and stability of gait and pain, fear, and quality of life (QOL). We hypothesized that individuals with CLBP would have greater gait variability and lower gait stability compared to healthy controls, and that these differences in trunk control would be increased in the daily living compared to in the laboratory. We hypothesized that changes in the trunk control of gait among participants with CLBP would show a relationship with pain intensity, fear of movement, and/ or QOL.

\section{Methods \\ Participants}

An investigation of gait in healthy participants and CLBP patients used $n=18$ as the sample size for each group, ${ }^{23}$ and a study of gaits in different environments used $n=19$ as the sample. ${ }^{18}$ Based on the effect size $\left(\eta_{\mathrm{p}}{ }^{2}=0.27\right)$ of a previous study, ${ }^{23}$ we calculated the necessary total sample size as 38 participants. We aimed for a minimum sample size of 20 in the present healthy control group and CLBP group. We recruited 20 healthy participants (mean age 56.75 \pm 9.43 years) from our laboratory's geographic region by using flyers distributed from September 2018 to March 2019, and 20 patients with CLBP (mean age $54.05 \pm 10.76$ years) from an orthopedic clinic from September 2018 to March 2020 in Figure 1. Healthy participants were included if they had no lower back pain (LBP) at enrollment and were excluded if they had any of the following: history of lumbar surgery, had received treatment for CLBP in the past 6 months, severely limited mobility, a progressive neurological disorder, or a terminal illness. The inclusion criteria for the CLBP patients were as follows: an LBP duration of $>3$ months; a score of $\geq 1$ on an 11-point numeric rating scale (NRS, 0-10) for pain intensity; and sufficient walking ability to travel to our laboratory without assistance. The exclusion criteria were as follows: previous spinal surgery (fusion, instrumentation, or discectomy), lumbar infiltration in the last 6 months, serious spinal pathology (cancer, inflammatory arthropathy, or acute vertebral fracture), spinal deformities, leg pain (eg, hip/knee OA), a diagnosis of neurological disease, and a stage of $\geq 2$ on a functional assessment staging test of Alzheimer's disease. The study protocol conformed to the Declaration of Helsinki. Before participating, each participant provided written informed consent. This study was approved by the Ethics Committee of Kio University Health Science Graduate School (approval no. H30-20). 


\section{Study Design}

For the experiment conducted in the laboratory environment, CLBP patients and healthy controls was asked to wear a single wearable tri-axial accelerometer (Axivity AX3, York, UK) on the fifth lumbar vertebra (L5). The participants received sufficient instruction on how to wear the device and practiced until they were able to wear it stably. The device was programmed to capture data at 100 $\mathrm{Hz}$ and at a range of $\pm 8 \mathrm{~g} .{ }^{24}$ Participants then walked for $>60 \mathrm{sec}$ at their preferred speed around an indoor track (30 m/loop). This laboratory-based gait measurement was then followed by gait measurement in a daily living environment while the participant was wearing the tri-axial accelerometer. For this part of the experiment, the participants were asked to wear tri-axial accelerometer for 3 days while performing their usual daily activities in their usual living environment; ${ }^{25}$ the participants removed the sensor during bathing and sleeping, and wore it at all other times.

After the measurement of gait in daily living was completed, each CLBP patient was instructed to fill out three questionnaires about their LBP over the 3 days of the experiment. The average pain intensity over time was assessed on an 11-point numerical rating scale (NRS: 0 $=$ no pain and $10=$ highest possible degree of pain). Kinesiophobia was assessed using the 11-item version of the Tampa Scale for Kinesiophobia in Japanese (TSK-11: $11=$ no fear of movement and $44=$ highest fear of movement). ${ }^{26}$ Physical disability caused by LBP was assessed by the Japanese version of the Roland-Morris Disability Questionnaire (RMDQ: $0=$ no physical disability and $24=$ worse physical function) ${ }^{27}$

\section{Data Processing and Analysis}

All data analyses related to the raw acceleration signals were performed using custom-made software designed on MATLAB R2019b (MathWorks, Natick, MA, USA). The mean wearing time for the tri-axial accelerometer was $907.10 \pm 21.44 \mathrm{~min} /$ day (range: $869-938$ ) for healthy controls and $903.35 \pm 19.98 \mathrm{~min} /$ day (range: 865-942) for CLBP patients, and all participants were included in the analyses because they wore the wearable sensor for a sufficient amount of time for the analyses. ${ }^{28}$ The algorithm and data segmentation techniques applied to the accelerometer data have been described in full. ${ }^{24,29}$ In brief, we subjected the vertical acceleration data to continuous wavelet transformation in order to estimate the initial contact and the final contact in the gait cycle. ${ }^{29}$
To ensure that the steady-state gait was analyzed, we removed the initial three steps and final three steps from the data captured over $60 \mathrm{sec}$. In the daily living environment, a mean of 15.75 walking epochs (range 6-32 epochs), each with a duration $>60 \mathrm{sec}$, was identified for each of the healthy participants. Similarly, a mean of 15.15 walking epochs (range 5-26), each with a duration $>60 \mathrm{sec}$, was identified for each of the CLBP patients. Prior to the calculation of additional variables, the acceleration signals were realigned to the Earth's gravitational constant, and a low-pass Butterworth filter with a cut-off frequency of $20 \mathrm{~Hz}$ was applied. ${ }^{30,31}$ The following variables were calculated using the accelerations in the anterior-posterior (AP) and mediallateral (ML) directions (see Figure 1A Figure 2A), since the directional control of movement is performed independently. ${ }^{32}$

\section{Trunk Variability of Gait}

The standard deviation (SD) is one of the classical linear estimates. To compute the stride-to-stride variability of gait, trunk acceleration during each stride was time-normalized (0-100\%) (see Figure 1B Figure 2B). At each of the 101 normalized time points, the SDs of the AP and ML trunk accelerations were calculated. Next, the average SD of these 101 SDs was calculated. ${ }^{11}$

Multiscale sample entropy (MSE) is a nonlinear parameter used to quantify the variability or the complexity of biological signals based on the dynamical system theory that the dynamics of motor behavior results from interactions among the nervous system, the body, and the environment in the performance of a particular task on multiple spatio-temporal scales ${ }^{12,13}$ (see Figure 1C Figure 2C). MSE is more sensitive than linear variability and can be used to investigate complexity in motor control. ${ }^{33}$ This index aims at quantifying the predictability of patterns: a time series containing many repetitive patterns (ie, one that is more predictable) would have relatively small MSE, whereas a less predictable process would have higher MSE and smaller regularity. ${ }^{12}$ In the present study, we used a range of $\tau$-values (1-6) to analyze entropy at different time scales while preserving the characteristics of the original time series. The length of sequences to be compared $(\mathrm{m})$ was fixed at 2, and the tolerance for accepting matches ( $r$ ) was fixed at $0.2 .^{34,35}$

\section{Trunk Stability of Gait}

The maximum Lyapunov exponent $\lambda$ (LyE) is a frequently used to quantify the dynamic stability of gait and the ability 
From September 2018

to March 2019
From September 2018 to March 2020

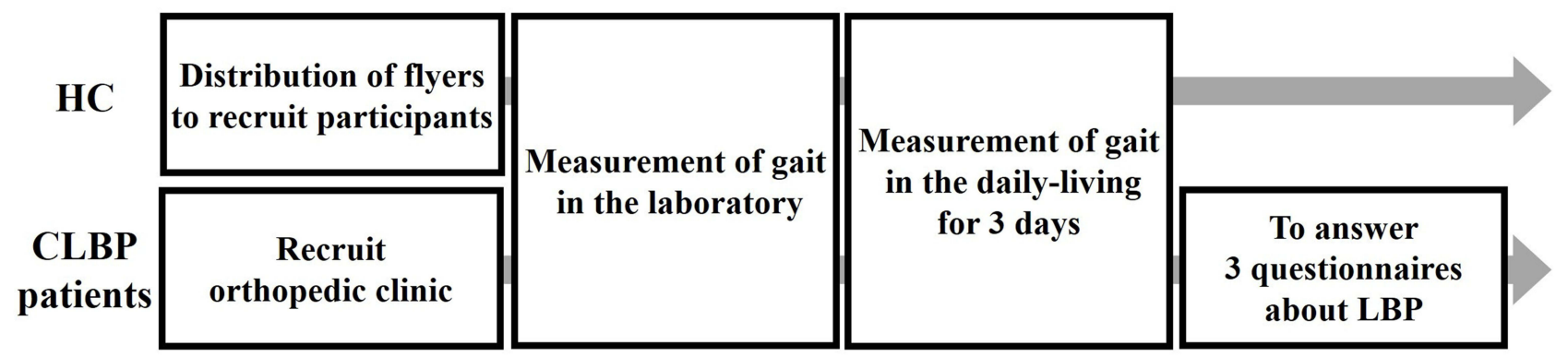

Figure I Flow diagram of the experimental procedures. Healthy controls (HCs) were recruited from our laboratory's geographic region using flyers distributed Sept. 2018 to March 2019, and chronic low back pain (CLBP) patients were recruited from an orthopedic clinic. HC and CLBP patients participated in this study from September 2018 to March 2020. The gait in daily-living environments was measured for 3 days. The CLBP patients answered questionnaires about their LBP over the 3 days when they wore a single wearable sensor.

of the motor system to attenuate small perturbations, defined as the divergence of trajectories in state space ${ }^{14}$ (see Figure 1D). The LyE thus measures the exponential rate of the divergence of trajectories of a state space constructed from kinematic data acquired from the gait. ${ }^{36}$ An inability of the motor system to diminish perturbations results in a higher divergence of the trajectories of state space and thus greater LyE values. Thus, the higher the LyE value, the lower the stability of the individual's gait. ${ }^{37,38}$

We calculated the LyE values using the method defined by Rosenstein et al. ${ }^{39}$ The state space reconstruction was composed of delay-embedded state spaces $(\mathrm{q}(\mathrm{t}), \mathrm{q}(\mathrm{t}+\tau), \mathrm{q}(\mathrm{t}+2 \tau) \ldots)$ of unfiltered ML and AP acceleration components. Time delays $(\tau)$ were calculated from the first minimum of the average mutual information function. An embedding dimension of $\mathrm{dE}=5$ was used for all data sets, as determined from a global falsenearest-neighbor analysis. The LyE was calculated as the slope of the logarithm of the average divergence across the span of $0-0.5$ strides as shown in Figure 2Figure 2D.

\section{Statistical Analyses}

We used the software program R (ver. 3.4.1) for all statistical analyses. Before conducting the statistical analyses, we confirmed that all data were normally distributed by using the Shapiro-Wilk normality test. We compared the age and gender distribution between the HC and CLBP groups by using the $t$-test and chi-squared test, respectively. For the examination of the trunk motor controls of gait, we performed a $2 \times 2$ [Environment (lab/daily living $) \times$ Group $(\mathrm{HC} / \mathrm{CLBP})]$ repeated measures analysis of variance (RM-ANOVA) in the ML and AP directions. Partial eta square $\left(\eta_{p}{ }^{2}\right)$ values were calculated for the identification of effect sizes. Independent and dependent $t$-tests were used for a post-hoc analysis when significant group by condition interactions were identified.

Independent $t$-tests were used to compare demographics between groups, and Cohen's d-values were calculated to indicate effect sizes. A commonly used interpretation is to refer to effect sizes as small $(\mathrm{d}=0.2$; $\left.\eta_{\mathrm{p}}{ }^{2}=0.01\right)$, medium $\left(\mathrm{d}=0.5 ; \eta_{\mathrm{p}}{ }^{2}=0.06\right)$, and large $(\mathrm{d}=$ $\left.0.8 ; \eta_{\mathrm{p}}{ }^{2}=0.14\right) .{ }^{40} \mathrm{We}$ evaluated the associations between the CLBP patients' trunk motor controls of gait and their symptoms by using Pearson product-moment correlation coefficients. The analysis was corrected for multiple comparisons using Holm corrections. ${ }^{41}$

Table I Participants' Characteristics and Comparison of Variables Between the HC and CLBP Groups

\begin{tabular}{|l|c|c|}
\hline & $\begin{array}{c}\text { Healthy Controls } \\
(\mathbf{H C})(\mathbf{n}=\mathbf{2 0})\end{array}$ & $\begin{array}{c}\text { Chronic Low Back Pain } \\
(\mathbf{C L B P})(\mathbf{n}=\mathbf{2 0})\end{array}$ \\
\hline Age (year) & $56.75 \pm 9.43$ & $54.05 \pm 10.76$ \\
\hline Gender & $\mathrm{M}=12, \mathrm{~F}=8$ & $\mathrm{M}=\mathrm{II}, \mathrm{F}=9$ \\
\hline Pain NRS & - & $4.40 \pm 1.32$ \\
\hline $\begin{array}{l}\text { Duration } \\
\text { (months) }\end{array}$ & - & $23.65 \pm 17.42$ \\
\hline TSK-II & - & $22.05 \pm 6.6 \mathrm{I}$ \\
\hline RMDQ & - & $4.60 \pm 2.91$ \\
\hline
\end{tabular}


A

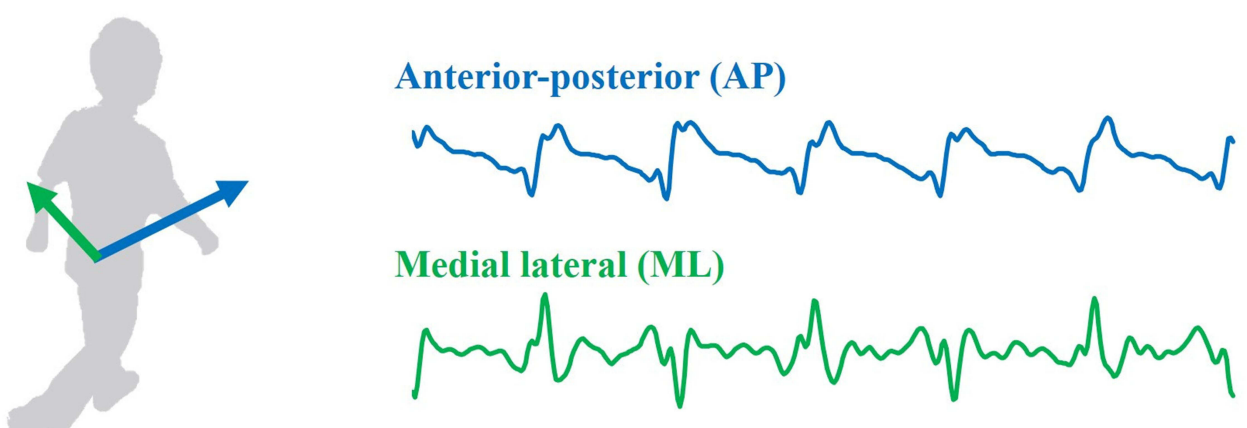

B

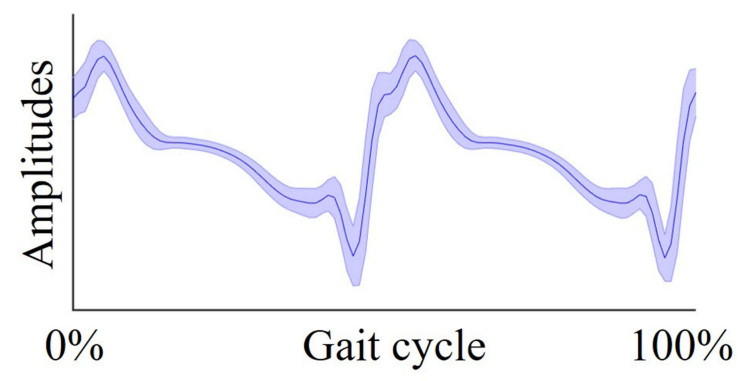

C Multiscale sample entropy (MSE)

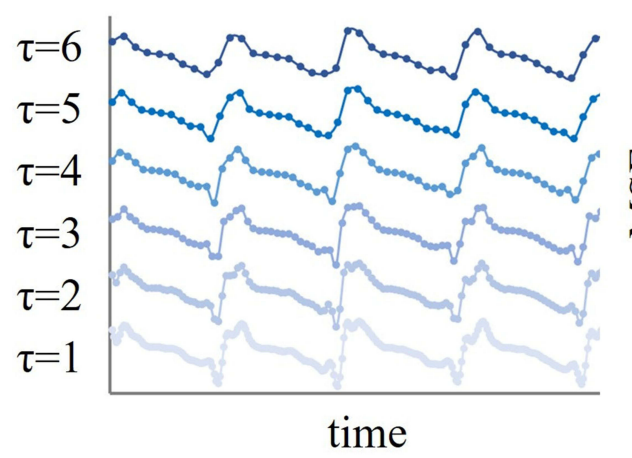

D Maximum lyapunov exponent (LyE)
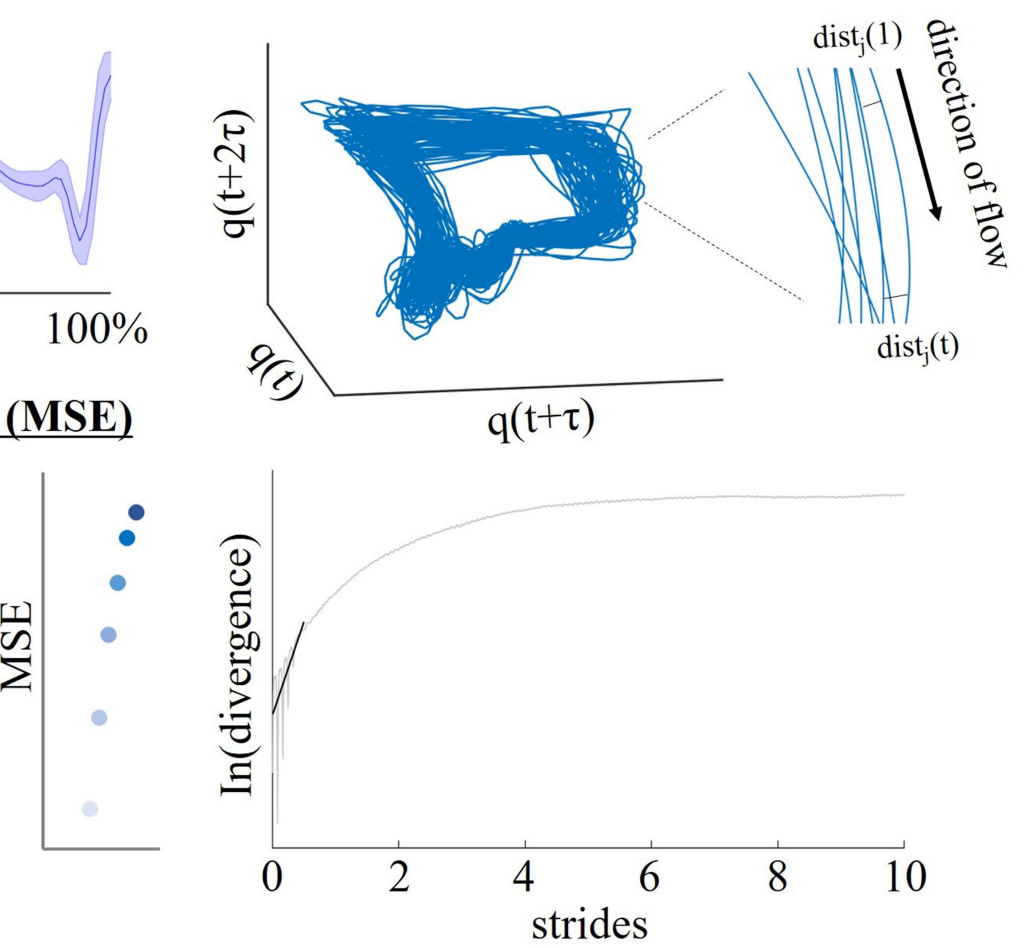

Figure 2 Calculation of gait parameters. (A) Direction of acceleration during gait. Blue line: anterior-posterior (AP). Green line: medial lateral (ML) direction. (B) Stride-tostride standard deviation (SD); x-axis, gait cycle (0-100\%); y-axis, amplitudes of AP direction. (C) Multiscale sample entropy (MSE). Time scales $\tau$ from I to 6 represent averages of a successively increasing number of data points in nonoverlapping windows $(\tau=I-6$ : from light blue to deep blue). The time series of $\tau=I$ shows original data. (D) The maximum Lyapunov exponent (LyE). A three-dimensional attractor (state space reconstruction of $q$ ) and close-up view of part of the attractor are shown. For each point on the attractor, the nearest neighbor was calculated, and divergence of these points was calculated as dist $j$ ( $t$ ). The average logarithmic rate of divergence was calculated to determine the LyEs.

\section{Results}

\section{Participant Characteristics in the $\mathrm{HC}$ and CLBP Groups}

Table 1 presents the demographic characteristics of the HC and CLBP groups and the symptoms of the CLBP group. No significant differences were observed in the characteristics of the HC and CLBP groups (age $p>0.05$ on independent samples $t$-test; gender $\mathrm{p}>0.05$ on chi-squared test).

\section{Trunk Variability of Gait}

Figure 3 provides the mean values and SDs for trunk variability and stability of gait in the HC and CLBP groups. Table 2 summarizes the results of the RMANOVA for trunk variability and stability of gait in both groups. The F-value, p-value, and partial eta square $\left(\eta_{\mathrm{p}}{ }^{2}\right)$ values in the RM-ANOVA are shown in Table 2. In the AP direction, the RM-ANOVA of the 
SD revealed significant main effects for Group but not for Environment. An interaction of Environment $\times$ Group was not significant. In the ML direction, the RM-ANOVA of the SD revealed significant main effects for Environment and for Group. An interaction of Environment $\times$ Group was not significant.

The RM-ANOVA of MSE revealed no significant main effects for Environment of Group in the AP direction, whereas in the ML direction, an MSE of $\tau$ $=1$ showed significant main effects for Group but not for Environment. An MSE of $\tau \geq 2$ showed significant main effects for Environment and for Group and revealed that the interaction of Environment $\times$ Group was significant.

The post hoc analyses were performed for MSE at $\tau \geq 2$ for ML and revealed that the occurrence of MSE at $\tau \geq 2$ in daily living was significantly higher than that in the lab for the CLBP group but not the HC group. In addition, MSE at $\tau \geq 4$ in the CLBP patients was significantly higher than that of the controls in the laboratory environment, and MSE at $\tau \geq 2$ in the CLBP group was significantly higher than that of the controls in the dailyliving environment.

\section{Trunk Stability of Gait}

In the AP direction, the LyE showed significant main effects for Environment and for Group. An interaction of Environment $\times$ Group was not significant. In the ML direction, the RM-ANOVA results for LyE revealed no significant main effects for Environment or Group. An interaction of Environment $\times$ Group was not significant. The post-hoc power analysis yielded a statistical power from 0.84 to 0.99 for RM-ANOVAs and 0.99 for the post hoc analysis of the RM-ANOVAs, confirming sufficient power.

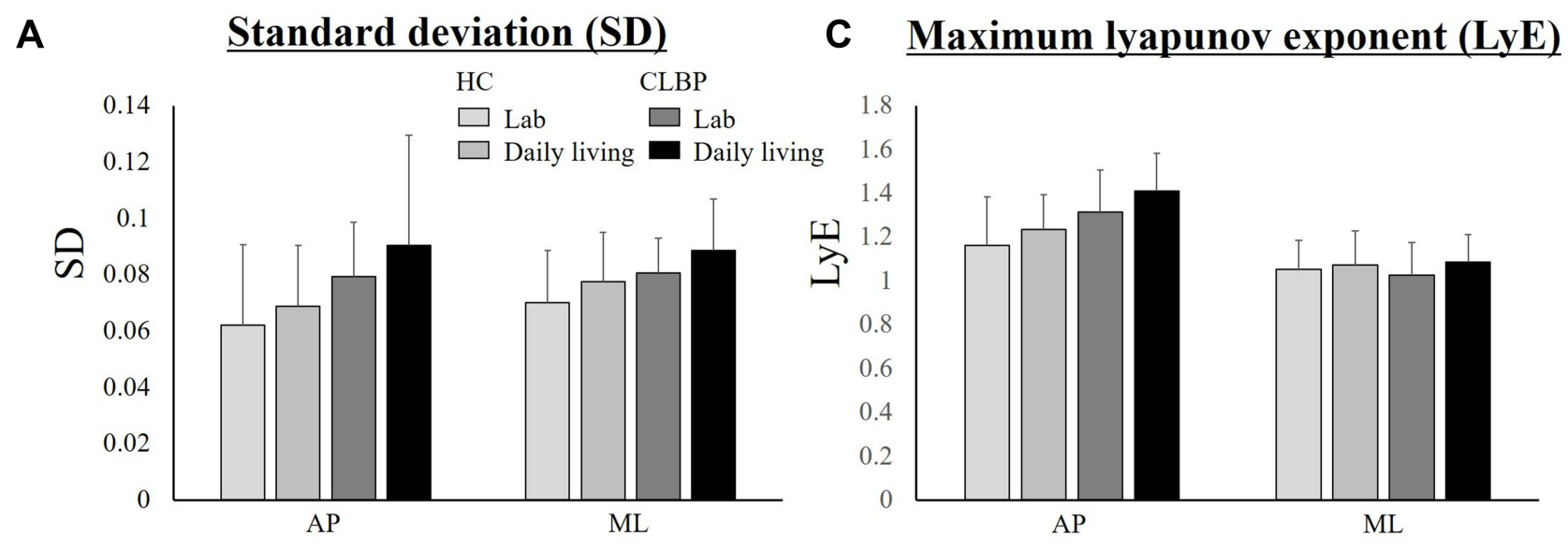

B

Multiscale sample entropy (MSE)
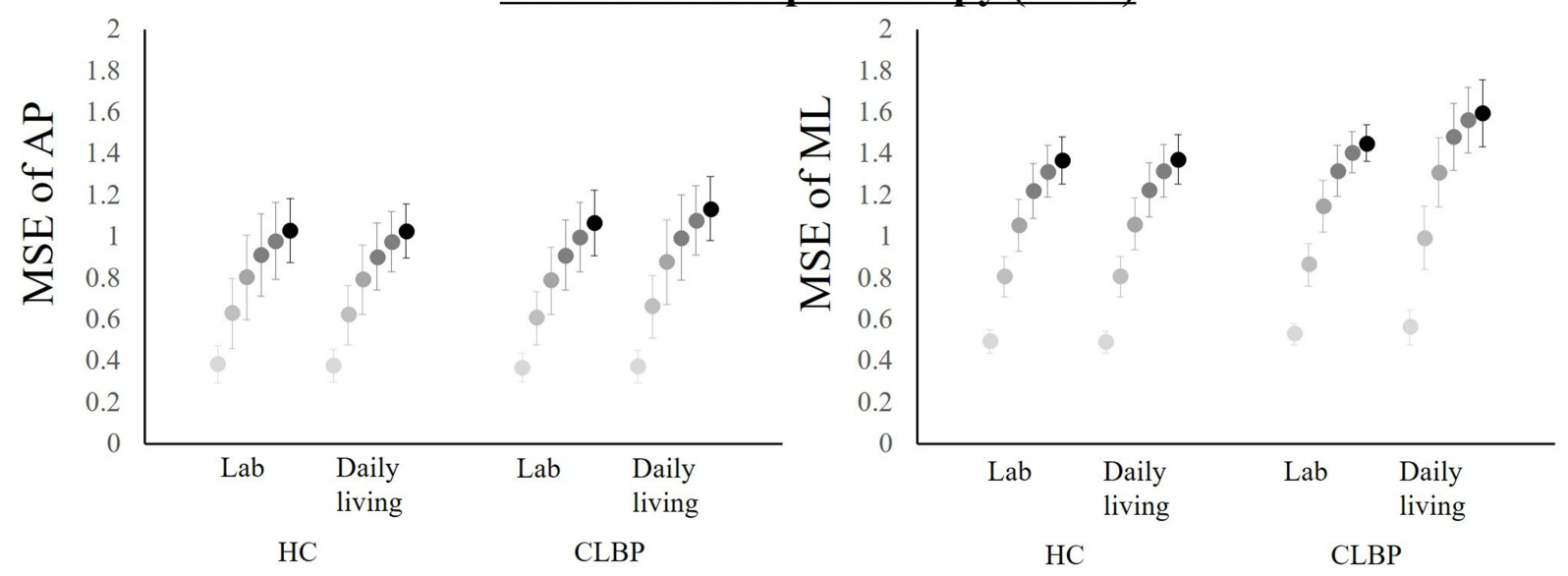

Figure 3 Trunk variability and stability of gait in laboratory and daily-living environments for the HC and CLBP groups. (A) Stride-to-stride SD, from left to right: laboratory and daily-living environments in the HC group, laboratory and daily-living in the CLBP group. (B) MSE. Time-scale $\tau$ values are represented by gray scale $(\tau=\mathrm{I}-6$ : from light gray to black). (C) The LyE. 


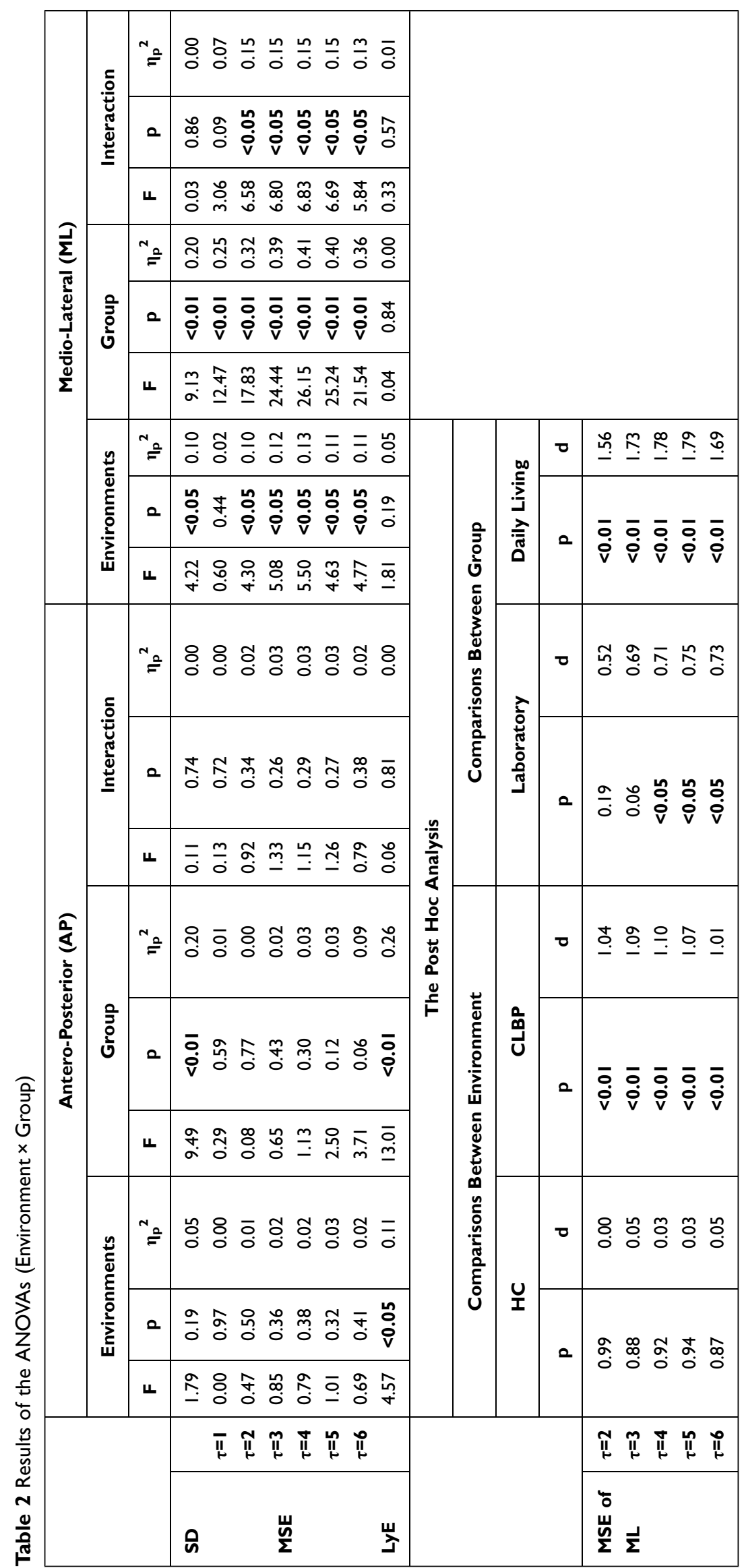




\section{The Associations Between the Trunk Motor Controls of Gait and the Questionnaires of the CLBP Patients}

The associations between the trunk motor controls of gait and pain intensity, the TSK-11, and the RMDQ are presented in Figure 4 . In the lab, the only significant correlation was between the MSE at $\tau=6$ of the ML direction and the TSK11. In daily living, the LyE of the AP direction was associated with both the TSK-11 and RMDQ responses, and the SD of the ML direction was associated with the TSK-11 responses. MSE at $\tau \geq 5$ in the ML direction was also associated with the pain-intensity NRS and TSK-11 results.

\section{Discussion}

A single wearable sensor placed on the lower back was used in this study to assess the trunk variability and stability of gait in individuals with chronic low back pain (CLBP) and healthy controls (HC) in laboratory and daily-living environments. We hypothesized that individuals with CLBP would have greater gait variability and lower gait stability compared to $\mathrm{HCs}$, and that these differences in trunk control would be greater in the daily- living environments than in the laboratory. We also hypothesized that differences in the trunk controls of gait would show a relationship to symptoms of CLBP.

Our results were as follows: (1) the standard deviation (SD) of trunk acceleration (ie, trunk variability) in the CLBP group was high regardless of the environment in the Anterior-Posterior (AP) direction and high but affected by the environment in the Medial-Lateral (ML) direction. (2) Multiscale sample entropy (MSE) (ie, complexity) was not affected by CLBP status or by the environment in the AP direction but was high in the CLBP group and affected by the environment in the ML direction. In addition, the MSE at $\tau \geq 2$ was higher in the CLBP group in the dailyliving environment. (3) The maximum Lyapunov Exponent (LyE) (ie, trunk stability) was high in the CLBP group and affected by the environment in the AP direction, but was not affected by CLBP status or environment in the ML direction. (4) These changes in trunk motor control due to CLBP were related to pain intensity, fear of movement, and/or deficits in activities of daily living (ADLs) in the daily-living environment but not the laboratory environment. All of the results of the RMANOVAs with significant differences showed medium or

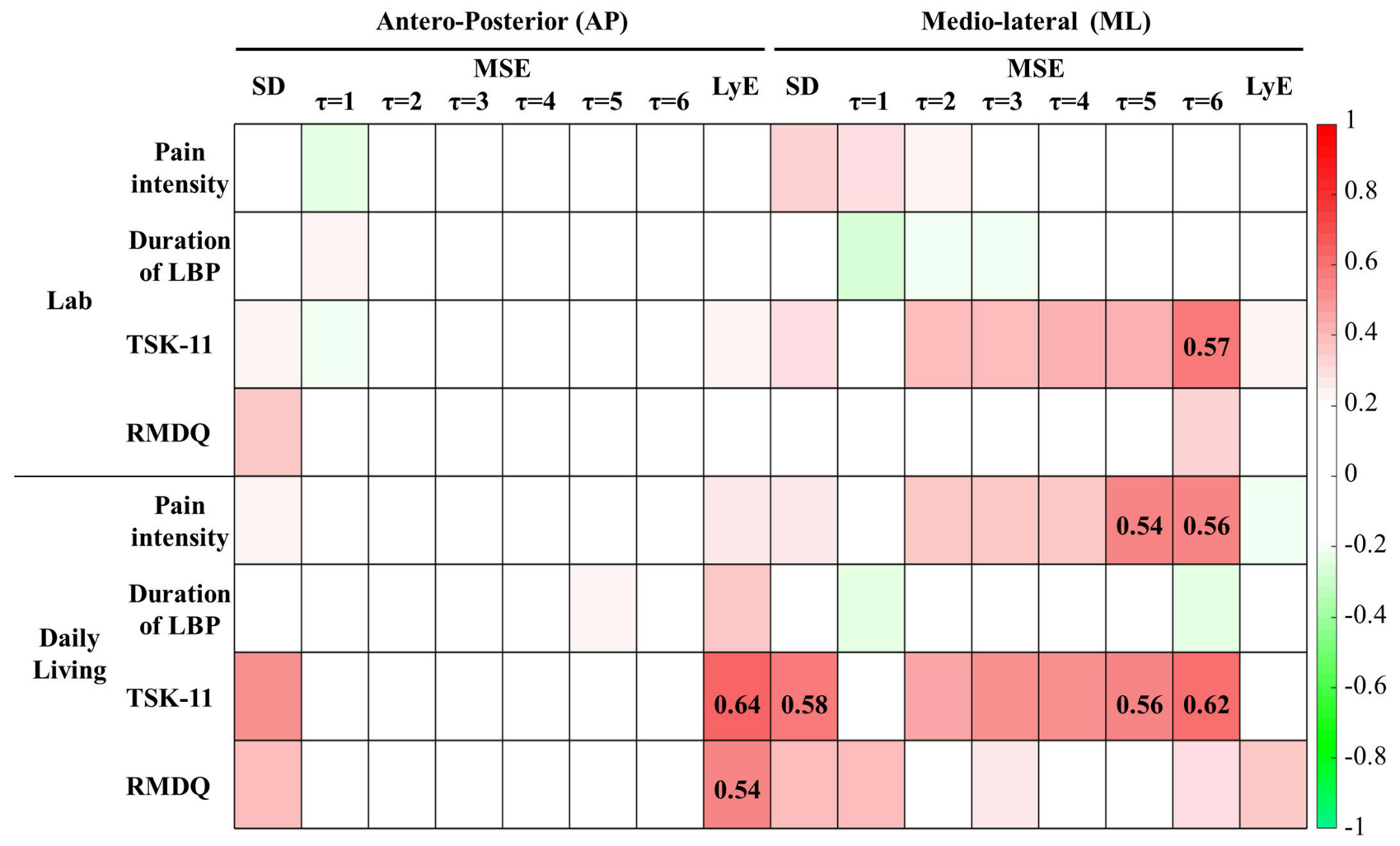

Figure 4 Heat map showing correlation coefficients between trunk motor controls of gait and symptoms of CLBP in the CLBP group. Darker pixels reflect higher correlation values (red: positive, green: negative). The $r$-value is indicated only in the pixels when the correlation was significant at $p<0.05$ using Holm corrections. 
large effect sizes. Overall, the results supported our hypothesis, but the AP and ML directions showed differences in these trends of trunk variability and stability of gait.

We compared TSK-11 and RMDQ with those obtained in previous studies, and we observed that TSK-11 and RMDQ were similar to reported values, and that the kinesiophobia and QOL of our CLBP patients were not deviant. ${ }^{42,43}$ In the present CLBP group, the mean score of kinesiophobia was 22.05. Kinesiophobia is described as a gradualizing syndrome but not a dichotomous characteristic. ${ }^{44}$ For the TSK11 , no cut-off value differentiating between high and low kinesiophobia has been reported; ${ }^{26}$ however, for the 17 -item TSK, the total score ranges from 17 to 68 and scores $>37$ are generally considered as reflecting a high level of kinesiophobia. $^{44,45}$ If we consider that $37(57 \%)$ on the 68 grade scale represents high kinesiophobia, it would be equal to 35 points on a 44-graded scale. In light of their TSK-11 scores, our participants appear to have suffered from slight kinesiophobia but not severe kinesiophobia. Our CLBP patients' mean score on the RMDQ was 4.60, and the reported cut-off point for the RMDQ is 2.5. ${ }^{46}$ Eighty percent of the present CLBP patients' scores were beyond this limit, and most of the CLBP patients had impaired QOL due to low back pain.

We also observed that the trunk variability of gait in the CLBP group was increased in the daily-living environment. Gait in the unstructured environment of daily living may be more difficult to predict and prone to perturbations compared to gait in structured clinical environments, because the unstructured environment reinforces the need to adapt movements with a high degree of freedom (DOF). From the perspective of spinal structure, the lumbar spine has the largest DOF number and therefore greater problems with controlling position, especially with high loads and in unpredictable environments. ${ }^{47}$ Loose control (ie, increased trunk variability) can cause LBP, as large displacements after trunk perturbations are associated with LBP, and failures of muscular control over the spine result in large tissue strains. ${ }^{48,49}$

The increased complexity of gait in CLBP that we observed in this study is consistent with the results of previous studies. ${ }^{50-52}$ Our present findings also revealed that the effect of environment or group was increased with time delay $\tau$, in agreement with previous research. ${ }^{53,54}$ In particular, a $\tau$-value $>4$ means that frequencies below $10-16 \mathrm{~Hz}$ contribute the most in characterizing the automaticity of the human gait. ${ }^{55}$
Our present findings demonstrated that the trunk variability of gait was related to pain intensity and kinesiophobia only in the daily-living environment. Other researchers reported that the automaticity of gait and variability were disrupted in relation to pain and/or fear, and that gait complexity was increased in patients with lumbar spinal stenosis; the latter phenomenon was described as being directly related to the intensity of low back pain. ${ }^{56,57}$ In addition, pain and fear each interfere with the neural control pathways for automaticity. ${ }^{58}$ Thus, changes in the neuromuscular control and/or pain or fear may compel participants to compensate for adjusting their gait pattern. From the above findings, we speculate that the unstructured environment of daily living, ie, unpredictable situations, may promote compensatory gait-loosening strategies and a lack of automaticity.

The trunk stability of gait is defined as the ability to maintain functional locomotion despite the presence of small kinematic disturbances or control errors. ${ }^{38}$ Individuals with CLBP have a deficiency in muscle coordination and reduced precision in the control of trunk movement due to diminished proprioceptive feedback. ${ }^{59}$ In addition, deficits of motor control are thought to be induced by pain or fear. ${ }^{60,61}$ These factors appear to cause disturbed control and recovery from perturbations and reduced stability. ${ }^{62,63}$ Some studies have described higher trunk variability and/or lower stability of gait in CLBP patients (as observed herein), but opposite results have also been reported in CLBP, describing decreased variability and/or increased stability of trunk movements due to increased trunk stiffness. ${ }^{4,64}$ Importantly, the studies that reported tight control of gait used a treadmill. Treadmill walking tends toward less variability and more stability compared to walking on solid ground or in daily-living environments. ${ }^{65}$ Although increased stiffness may contribute to the control of smallamplitude perturbations experienced in the predictable environment of treadmill walking, it may limit the potential for control and recovery from larger perturbations. ${ }^{62}$ Thus, CLBP patients' lumbar movement during gait may be unstable due to an inability to adapt to perturbations in the unstructured environment of daily living by pathological kinematic disturbances or control errors.

Our present analyses revealed that for both stability and variability of gait, the data in the AP and ML directions showed different trends. Specifically, in the AP direction, stability of gait was impaired at medium effect sizes, and the variability was not affected by environmental changes; in the ML direction, the variability was affected 
by the environment and by CLBP at medium or large effect sizes, but the stability was maintained. Changes in the trunk variability of gait were reported to occur as an adaptation to the environment to maintain trunk stability. ${ }^{18}$ A decrease in stability in the AP direction, even though the variability does not change, may indicate less compensation or inadequate compensation. ${ }^{33}$ Dysfunction of the agonist/antagonist relationship in lumbar extension also impedes the motor adaptation of perturbation. ${ }^{66,67}$ Therefore, in the AP direction, individuals with CLBP may have difficulty adapting to the environment, resulting in decreased stability in unstructured daily living. In contrast, in the ML direction, the stability of gait can be maintained by adaptations to the environment via changes in variability. These results were associated with the patients' subjective intensity of pain and fear of movement, and they were more pronounced in daily living. Our results also demonstrated that the trunk stability of gait in the AP direction was related to the patients' physical disability in daily living. Other studies have shown that pain and/or fear promote changes in the sensory-motor control system and movement strategy. ${ }^{68,69}$ Changes in gait control modified by pain and/or fear of movement may disturb a person's adaptation to their environment.

The present study has limitations to address. First, because the sample size was relatively small ( $\mathrm{n}=20$ for each group), the results should be interpreted with caution and their generalizability remains unclear. Future studies with a larger sample size are needed to confirm these findings. Second, because the questionnaires about pain and fear of movement were not assessed separately in the laboratory and daily living, the questionnaire responses may have tended to reflect the pain experienced in the daily-living environment. Third, we did not obtain the participants' home layouts as their daily-living environments. Our results, which showed that the trunk variability and stability of gait are related to pain and fear, support the concept that environmental adaptation is disturbed by chronic low back pain. Fourth, we compared gait parameters between one epoch in the laboratory environment and multiple epochs in the daily-living environment. By extracting epochs longer than 60 seconds, a statistically precise estimate of gait control is possible. We also considered that repeatable data were obtained because the gait data were recorded using the same environment for all participants (ie, a 30-m-loop indoor track). Unlike the laboratory environment, since the environments in each epoch daily-living environments (eg, location and route) were not constant, we used the average value of the epochs as the index of gait in the daily-living environments.
However, the comparison of these environments may have been statistically insufficient.

In conclusion, the present results revealed that CLBP patients undergo changes in trunk variability and stability of gait depending on the environment, and these changes are related to pain, fear, and quality of life. Interestingly, in the AP direction, the stability of gait was impaired, and the variability was not affected by environmental changes; in the ML direction, the variability was affected by the environment, but the stability was maintained. Although the underlying causes of these different adaptations of gait variability and stability by direction remain to be investigated, we suspect that changes in the muscle and/or sensory-motor control system are responsible. Our findings demonstrate the utility of an accelerometer-based assessment of qualitative gait in the daily living of CLBP patients, as it provides information that is unavailable in a general practice setting. Our results also suggest that the changes in the lumbar control of gait in a daily-living environment are more pronounced than those in a laboratory environment.

\section{Funding}

This research was not supported by a grant.

\section{Disclosure}

The authors have no conflicts of interest to declare.

\section{References}

1. Marshall LM, Litwack-Harrison S, Cawthon PM, et al. A prospective study of back pain and risk of falls among older community-dwelling women. J Gerontol Ser A Biol Sci Med Sci. 2016;71(9):1177-1183. doi:10.1093/gerona/glv225

2. Marshall LM, Litwack-Harrison S, Makris UE, et al. A prospective study of back pain and risk of falls among older community-dwelling men. J Gerontol A Biol Sci Med Sci. 2017;72(9):1264-1269. doi:10.1093/gerona/glw227

3. Vos T, Abajobir AA, Abbafati C, et al. Global, regional, and national incidence, prevalence, and years lived with disability for 328 diseases and injuries for 195 countries, 1990-2016: a systematic analysis for the Global Burden of Disease Study 2016. Lancet. 2017;390 (10100):1211-1259. doi:10.1016/S0140-6736(17)32154-2

4. Lamoth CJC, Meijer OG, Daffertshofer A, Wuisman PIJM, Beek PJ. Effects of chronic low back pain on trunk coordination and back muscle activity during walking: changes in motor control. Eur Spine J. 2006;15(1):23-40. doi:10.1007/s00586-004-0825-y

5. Hamacher D, Hamacher D, Schega L. A cognitive dual task affects gait variability in patients suffering from chronic low back pain. Exp Brain Res. 2014;232(11):3509-3513. doi:10.1007/s00221-014-4 039-1

6. Hamacher D, Hamacher D, Herold F, Schega L. Are there differences in the dual-task walking variability of minimum toe clearance in chronic low back pain patients and healthy controls? Gait Posture. 2016;49:97-101. doi:10.1016/j.gaitpost.2016.06.026 
7. Koch C, Hänsel F. Chronic non-specific low back pain and motor control during gait. Front Psychol. 2018;9:1-8. doi:10.3389/ fpsyg.2018.02236

8. Laird RA, Gilbert J, Kent P, Keating JL. Comparing lumbo-pelvic kinematics in people with and without back pain: a systematic review and meta-analysis. BMC Musculoskelet Disord. 2014;15(1):1-13. doi:10.1186/1471-2474-15-229

9. Hodges PW. Pain and motor control: from the laboratory to rehabilitation. J Electromyogr Kinesiol. 2011;21(2):220-228. doi:10.1016/j.jelekin.2011.01.002

10. Bruijn SM, Meijer OG, Beek PJ, Van Dieen JH. Assessing the stability of human locomotion: a review of current measures. J R Soc Interface. 2013;10(83):20120999. doi:10.1098/rsif.2012.0999

11. Magnani RM, Lehnen GC, Rodrigues FB, de Sá e Souza GS, de Oliveira Andrade A, Vieira MF. Local dynamic stability and gait variability during attentional tasks in young adults. Gait Posture. 2017;55:105-108. doi:10.1016/j.gaitpost.2017.04.019

12. Costa M, Peng CK, Goldberger AL, Hausdorff JM. Multiscale entropy analysis of human gait dynamics. Phys A Stat Mech Appl. 2003;330(1-2):53-60. doi:10.1016/j.physa.2003.08.022

13. Costa M, Goldberger AL, Peng CK. Multiscale entropy analysis of biological signals. Phys Rev E - Stat Nonlinear, Soft Matter Phys. 2005;71(2):1-18. doi:10.1103/PhysRevE.71.021906

14. Kang HG, Dingwell JB. Intra-session reliability of local dynamic stability of walking. Gait Posture. 2006;24(3):386-390. doi:10.1016/j.gaitpost.2005.11.004

15. Kao PC, Dingwell JB, Higginson JS, Binder-Macleod S. Dynamic instability during post-stroke hemiparetic walking. Gait Posture. 2014;40(3):457-463. doi:10.1016/j.gaitpost.2014.05.014

16. Ihlen EAF, Weiss A, Bourke A, Helbostad JL, Hausdorff JM. The complexity of daily life walking in older adult community-dwelling fallers and non-fallers. $J$ Biomech. 2016;49(9):1420-1428. doi:10.1016/j.jbiomech.2016.02.055

17. Ihlen EAF, van Schooten KS, Bruijn SM, Pijnappels M, van Dieën JH. Fractional stability of trunk acceleration dynamics of daily-life walking: toward a unified concept of gait stability. Front Physiol. 2017;8(AUG):1-15. doi:10.3389/fphys.2017.00516

18. Tamburini P, Storm F, Buckley C, Bisi MC, Stagni R, Mazzà C. Moving from laboratory to real life conditions: influence on the assessment of variability and stability of gait. Gait Posture. 2018;59:248-252. doi:10.1016/j.gaitpost.2017.10.024

19. De Bruin ED, Hubli M, Hofer P, Wolf P, Murer K, Zijlstra W. Validity and reliability of accelerometer-based gait assessment in patients with diabetes on challenging surfaces. $J$ Aging Res. 2012;2012:1-9. doi:10.1155/2012/954378

20. Lord S, Galna B, Rochester L. Moving forward on gait measurement: toward a more refined approach. Mov Disord. 2013;28 (11):1534-1543. doi:10.1002/mds.25545

21. Weiss A, Brozgol M, Dorfman M, et al. Does the evaluation of gait quality during daily life provide insight into fall risk? A novel approach using 3-Day accelerometer recordings. Neurorehabil Neural Repair. 2013;27(8):742-752. doi:10.1177/1545968313491004

22. Weiss A, Herman T, Giladi N, Hausdorff JM. Objective assessment of fall risk in Parkinson's disease using a body-fixed sensor worn for 3 days. PLoS One. 2014;9(5):e96675. doi:10.1371/journal. pone.0096675

23. Gombatto SP, Brock T, DeLork A, Jones G, Madden E, Rinere C. Lumbar spine kinematics during walking in people with and people without low back pain. Gait Posture. 2015;42(4):539-544. doi:10.1016/j.gaitpost.2015.08.010

24. Del Din S, Godfrey A, Rochester L. Validation of an accelerometer to quantify a comprehensive battery of gait characteristics in healthy older adults and Parkinson's disease: toward clinical and at home use. IEEE J Biomed Heal Informatics. 2016;20(3):838-847. doi:10.1109/ JBHI.2015.2419317
25. Van Schooten KS, Rispens SM, Elders PJM, Lips P, Van Dieën JH, Pijnappels M. Assessing physical activity in older adults: required days of trunk accelerometer measurements for reliable estimation. J Aging Phys Act. 2015;23(1):9-17. doi:10.1123/JAPA.2013-0103

26. Woby SR, Roach NK, Urmston M, Watson PJ. Psychometric properties of the TSK-11: a shortened version of the Tampa Scale for Kinesiophobia. Pain. 2005;117(1-2):137-144. doi:10.1016/j. pain.2005.05.029

27. Roland MO. The natural history of back pain. Practitioner. 1983;227 (1381):1119-1122.

28. Choi L, Liu Z, Matthews CE, Buchowski MS. Validation of accelerometer wear and nonwear time classification algorithm. Med Sci Sport Exerc. 2011;43(2):357-364. doi:10.1249/ MSS.0b013e3181ed61a3

29. McCamley J, Donati M, Grimpampi E, Mazzà C. An enhanced estimate of initial contact and final contact instants of time using lower trunk inertial sensor data. Gait Posture. 2012;36(2):316-318. doi:10.1016/j.gaitpost.2012.02.019

30. Buckley C, Galna B, Rochester L, Mazzà C. Quantification of upper body movements during gait in older adults and in those with Parkinson's disease: impact of acceleration realignment methodologies. Gait Posture. 2017;52:265-271. doi:10.1016/j. gaitpost.2016.11.047

31. Moe-Nilssen R. A new method for evaluating motor control in gait under real-life environmental conditions. Part 2: gait analysis. Clin Biomech. 1998;13(4-5):328-335. doi:10.1016/S0268-0033(98) 00090-4

32. Bruijn SM, Van Dieën JH. Control of human gait stability through foot placement. $J R$ Soc Interface. 2018;15(143):20170816. doi:10.1098/rsif.2017.0816

33. Stergiou N, Decker LM. Human movement variability, nonlinear dynamics, and pathology: is there a connection? Hum Mov Sci. 2011;30(5):869-888. doi:10.1016/j.humov.2011.06.002

34. Pincus SM. Approximate entropy as a measure of system complexity. Proc Natl Acad Sci USA. 1991;88(6):2297-2301. doi:10.1073/ pnas.88.6.2297

35. Richman JS, Moorman JR. Physiological time-series analysis using approximate entropy and sample entropy. Am J Physiol Hear Circ Physiol. 2000;278(6):H2039-H2049. doi:10.1152/ ajpheart.2000.278.6.H2039.

36. Dingwell JB, Marin LC. Kinematic variability and local dynamic stability of upper body motions when walking at different speeds. J Biomech. 2006;39(3):444-452. doi:10.1016/j.jbiomech.2004.12.014

37. Mehdizadeh S. The largest Lyapunov exponent of gait in young and elderly individuals: a systematic review. Gait Posture. 2018;60:241-250. doi:10.1016/j.gaitpost.2017.12.016

38. England SA, Granata KP. The influence of gait speed on local dynamic stability of walking. Gait Posture. 2007;25(2):172-178. doi:10.1016/j.gaitpost.2006.03.003

39. Rosenstein MT, Collins JJ, De Luca CJ. A practical method for calculating largest Lyapunov exponents from small data sets. Phys D Nonlinear Phenom. 1993;65(1-2):117-134. doi:10.1016/01672789(93)90009-P

40. Cohen J. Statistical Power Analysis for the Behavioral Sciences. Routledge; 1988.

41. Holm S. Board of the foundation of the scandinavian journal of statistics. Scand J Stat. 1979;6:65-70.

42. Crosbie J, De Faria Negrão Filho R, Nascimento DP, Ferreira P. Coordination of spinal motion in the transverse and frontal planes during walking in people with and without recurrent low back pain. Spine. 2013;38(5):E286-E292. doi:10.1097/BRS.0b013e318281de28

43. Larsson C, Ekvall Hansson E, Sundquist K, Jakobsson U. Kinesiophobia and its relation to pain characteristics and cognitive affective variables in older adults with chronic pain. BMC Geriatr. 2016;16(1):1-7. doi:10.1186/s12877-016-0302-6 
44. Ejcm S-M, Swinkels RAHM, Verbeek ALM, Vlaeyen JWS, Oostendorp RAB. Psychometric properties of the Tampa Scale for kinesiophobia and the fear-avoidance beliefs questionnaire in acute low back pain. Man Ther. 2003;8(1):29-36. doi:10.1054/math.2002.0484

45. Vlaeyen JWS, Amj K-S, Rotteveel AM, Ruesink R, Heuts PHTG. The role of fear of movement/(re)injury in pain disability. $J$ Occup Rehabil. 1995;5(4):235-252. doi:10.1007/BF02109988

46. Monticone M, Baiardi P, Vanti C, et al. Responsiveness of the oswestry disability index and the roland morris disability Questionnaire in Italian subjects with sub-acute and chronic low back pain. Eur Spine J. 2012;21(1):122-129. doi:10.1007/s00586011-1959-3

47. Panjabi MM. The stabilizing system of the spine: part I. function, dysfunction, adaptation, and enhancement. J Spinal Disord. 1992;5 (4):383-389. doi:10.1097/00002517-199212000-00001

48. Cholewicki J, Silfies SP, Shah RA, et al. Delayed trunk muscle reflex responses increase the risk of low back injuries. Spine. 2005;30 (23):2614-2620. doi:10.1097/01.brs.0000188273.27463.bc

49. Van Dieën JH, Peter Reeves N, Kawchuk G, Van Dillen LR, Hodges PW. Motor control changes in low back pain: divergence in presentations and mechanisms. J Orthop Sports Phys Ther. 2019;49 (6):370-379. doi:10.2519/jospt.2019.7917

50. Arif M, Ohtaki Y, Nagatomi R. Estimation of the effect of cadence on gait stability in young and elderly people using approximate entropy technique. Meas Sci. 2004;4:29-40.

51. Karmakar CK, Khandoker AH, Begg RK, Palaniswami M, Taylor S Understanding ageing effects by approximate entropy analysis of gait variability. In: 2007 29th Annual International Conference of the IEEE Engineering in Medicine and Biology Society; 2007; IEEE. 1965-1968. doi:10.1109/IEMBS.2007.4352703.

52. Lamoth CJC, Ainsworth E, Polomski W, Houdijk H. Variability and stability analysis of walking of transfemoral amputees. Med Eng Phys. 2010;32(9):1009-1014. doi:10.1016/j.medengphy.2010.07.001

53. Bisi MC, Riva F, Stagni R. Measures of gait stability: performance on adults and toddlers at the beginning of independent walking. $J$ Neuroeng Rehabil. 2014;11(1):1-9. doi:10.1186/1743-0003-11-131

54. Riva F, Toebes MJP, Pijnappels M, Stagni R, van Dieën JH. Estimating fall risk with inertial sensors using gait stability measures that do not require step detection. Gait Posture. 2013;38(2):170-174. doi:10.1016/j.gaitpost.2013.05.002

55. Bisi MC, Stagni R. Complexity of human gait pattern at different ages assessed using multiscale entropy: from development to decline. Gait Posture. 2016;47:37-42. doi:10.1016/j.gaitpost.2016.04.001

56. Papadakis NC, Christakis DG, Tzagarakis GN, et al. Gait variability measurements in lumbar spinal stenosis patients: part A. Comparison with healthy subjects. Physiol Meas. 2009;30(11):1171-1186. doi:10.1088/0967-3334/30/11/003

57. Clark DJ. Automaticity of walking: functional significance, mechanisms, measurement and rehabilitation strategies. Front Hum Neurosci. 2015;9(MAY):1-13. doi:10.3389/fnhum.2015.00246
58. Farina S, Tinazzi M, Le Pera D, Valeriani M. Pain-related modulation of the human motor cortex. Neurol Res. 2003;25(2):130-142. doi:10.1179/016164103101201283

59. Tong MH, Mousavi SJ, Kiers H, Ferreira P, Refshauge K, van Dieën J. Is there a relationship between lumbar proprioception and low back pain? A systematic review with meta-analysis. Arch Phys Med Rehabil. 2017;98(1):120-136.e2. doi:10.1016/j.apmr.2016. 05.016

60. Langevin HM, Sherman KJ. Pathophysiological model for chronic low back pain integrating connective tissue and nervous system mechanisms. Med Hypotheses. 2007;68(1):74-80. doi:10.1016/j. mehy.2006.06.033

61. Tucker K, Larsson AK, Oknelid S, Hodges P. Similar alteration of motor unit recruitment strategies during the anticipation and experience of pain. Pain. 2012;153(3):636-643. doi:10.1016/j. pain.2011.11.024

62. Mok NW, Hodges PW. Movement of the lumbar spine is critical for maintenance of postural recovery following support surface perturbation. Exp Brain Res. 2013;231(3):305-313. doi:10.1007/ s00221-013-3692-0

63. Ross GB, Mavor M, Brown SHM, Graham RB. The effects of experimentally induced low back pain on spine rotational stiffness and local dynamic stability. Ann Biomed Eng. 2015;43(9):2120-2130. doi:10.1007/s10439-015-1268-9

64. van den Hoorn W, Hug F, Hodges PW, Bruijn SM, van Dieën JH. Effects of noxious stimulation to the back or calf muscles on gait stability. $J$ Biomech. 2015;48(15):4109-4115. doi:10.1016/j. jbiomech.2015.10.013

65. Dingwell JB, Cusumano JP, Cavanagh PR, Sternad D. Local dynamic stability versus kinematic variability of continuous overground and treadmill walking. J Biomech Eng. 2001;123(1):27-32. doi:10.1115/ 1.1336798

66. Vogt L, Pfeifer K, Banzer W. Neuromuscular control of walking with chronic low-back pain. Man Ther. 2003;8(1):21-28. doi:10.1054/ math.2002.0476

67. Ghamkhar L, Kahlaee AH. Trunk muscles activation pattern during walking in subjects with and without chronic low back pain: a systematic review. PMR. 2015;7(5):519-526. doi:10.1016/j. pmrj.2015.01.013

68. Karos K, Meulders A, Gatzounis R, Seelen HAM, Geers RPG, Vlaeyen JWS. Fear of pain changes movement: motor behaviour following the acquisition of pain-related fear. Eur J Pain. 2017;21 (8):1432-1442. doi:10.1002/ejp.1044

69. Nishi Y, Osumi M, Nobusako S, Takeda K, Morioka S. Avoidance behavioral difference in acquisition and extinction of pain-related fear. Front Behav Neurosci. 2019;13:1-10. doi:10.3389/fnbeh.2019. 00236
Journal of Pain Research

\section{Publish your work in this journal}

The Journal of Pain Research is an international, peer reviewed, open access, online journal that welcomes laboratory and clinical findings in the fields of pain research and the prevention and management of pain. Original research, reviews, symposium reports, hypothesis formation and commentaries are all considered for publication. The manuscript management system is completely online and includes a very quick and fair peer-review system, which is all easy to use. Visit http:// www.dovepress.com/testimonials.php to read real quotes from published authors. 